\title{
An Electronic-Nose Sensor Node Based on a Polymer-Coated Surface Acoustic Wave Array for Wireless Sensor Network Applications
}

\author{
Kea-Tiong Tang *, Cheng-Han Li and Shih-Wen Chiu \\ Department of Electrical Engineering, National Tsing Hua University, No. 101, Sec. 2, Kuang-Fu \\ Road, Hsinchu 30013, Taiwan; E-Mails: chli@larc.ee.nthu.edu.tw (C.-H.L.); \\ swchiu@larc.ee.nthu.edu.tw (S.-W.C.) \\ * Author to whom correspondence should be addressed; E-Mail: kttang@ee.nthu.edu.tw; \\ Tel.: +886-3-516-2178; Fax: +886-3-571-5971.
}

Received: 3 February 2011; in revised form: 17 February 2011 / Accepted: 7 April 2011 / Published: 28 April 2011

\begin{abstract}
This study developed an electronic-nose sensor node based on a polymer-coated surface acoustic wave (SAW) sensor array. The sensor node comprised an SAW sensor array, a frequency readout circuit, and an Octopus II wireless module. The sensor array was fabricated on a large $K^{2} 128^{\circ}$ YX LiNbO3 sensing substrate. On the surface of this substrate, an interdigital transducer (IDT) was produced with a $\mathrm{Cr} / \mathrm{Au}$ film as its metallic structure. A mixed-mode frequency readout application specific integrated circuit (ASIC) was fabricated using a TSMC $0.18 \mu \mathrm{m}$ process. The ASIC output was connected to a wireless module to transmit sensor data to a base station for data storage and analysis. This sensor node is applicable for wireless sensor network (WSN) applications.
\end{abstract}

Keywords: electronic nose; wireless sensor network; sensor node; SAW sensor array

\section{Introduction}

Wireless sensor networks (WSNs) [1,2] comprise a number of light-weight low-power sensor nodes. Each node is equipped with a number of sensors, interface electronics, and a wireless communication module to deliver the collected data. Applications of WSNs have been proposed in areas as diverse as health care [3], monitoring of industrial equipment [4], precision horticulture [5], volcanology [6], habitat observation [7] and monitoring of engines [8]. Issues associated with air pollution have been 
attracting increased attention because air quality has a direct influence on human health. Combining gas sensors within a WSN is an effective means to monitor urban air pollution [9]. However, it has proven unfeasible for general applications because each gas sensor has a corresponding target gas, requiring the installation of numerous sensors to monitor multiple gases. Electronic nose (E-Nose) systems have shown great potential in odor detection, analysis, and recognition. E-Noses first appeared in 1987, and were designed to mimic mammalian olfactory systems [10]. Olfactory processes do not perceive odors using only a single receptor, but rather detect and recognize scents according to an array of multiple receptors, with each combination representing a different odor. The permutations and combinations enable the recognition of complex gasses such as volatile organic compounds (VOCs) [11,12] and the odor of fruits [13]. Incorporating a WSN with an E-Nose to form a sensor array would greatly expand the scope of odor classification and practical applicability.

A polymer-coated surface acoustic wave (SAW) array is one of the best choices to achieve high sensitivity in applications requiring the detection of organic gasses [14,15]. Currently SAW devices are being used for a variety of chemical applications because of their high sensitivity, fully reversible behavior, and high signal-to-noise ratio [16]. For example, piezoelectric substrates are used to transform energy from mechanical strain to electric signals. Interdigital transducers (IDTs) are input and output comb-like metal electrodes, used as energy transformation structures on the surface of selected substrates. When an AC voltage is applied to an input IDT, dynamic strain is induced, launching a wave across the surface of the substrate. The induced surface wave propagates through the active sensing region, to be received and transformed into electrical signals by the output IDT. A change in mass on the active sensing region can be detected, according to changes in magnitude and phase shift in the AC signal between the input and output IDTs. To increase the selectivity and sensitivity of the sensor, a variety of polymers are used to coat the active sensing region of the SAW sensors to absorb molecules of the target gas. The traditional approach to building up a SAW-based E-Nose system has been to connect the SAW array to a spectrum analyzer or a frequency counter to monitor frequency shifts in the SAW devices. Unfortunately, neither of these options is practical for portable or WSN applications, due to their bulk and high price. To resolve this problem, a small, inexpensive frequency readout scheme is required.

Fabricating frequency readout circuitry using integrated circuits (IC) not only reduces the size of the system, but also the cost of mass production [17]. In recent years, designers have had the option to integrate general WSN platforms [18,19] with sensor devices for a variety of applications. In this study, we report an efficient E-Nose sensor node comprising a $2 \times 2$ non-continuous chemical SAW sensor array chip using MEMS technology self-assembled with polymer coatings on the active sensing region [20,21], application specific integrated circuit (ASIC) chip for a mixed signal interface, and a WSN platform (Octopus II). The low-power, high-resolution ASIC chip was fabricated using a TSMC $0.18 \mu \mathrm{m}$ 1P6M standard CMOS process. The ASIC chip was connected directly to an array of four SAW sensors, outputting frequency data representative of the sensor response. The output data was transmitted to a base station using an Octopus II wireless module. Results of measuring the chip and experimental data are presented in the following sections.

In Section 2, we introduce the proposed E-Nose gas sensor node and the experimental setup. In Section 3, we show the experimental results of the SAW sensor, the ASIC interface, and the fabricated sensor node. A brief conclusion is provided in Section 4. 


\section{Proposed E-Nose Sensor Node}

Figure 1 presents a block diagram of the proposed E-Nose sensor node. The Sensor node comprises three major parts: an SAW sensor array, a mixed signal ASIC chip, and a WSN platform (Octopus II).

Figure 1. Block diagram of SAW sensor node.

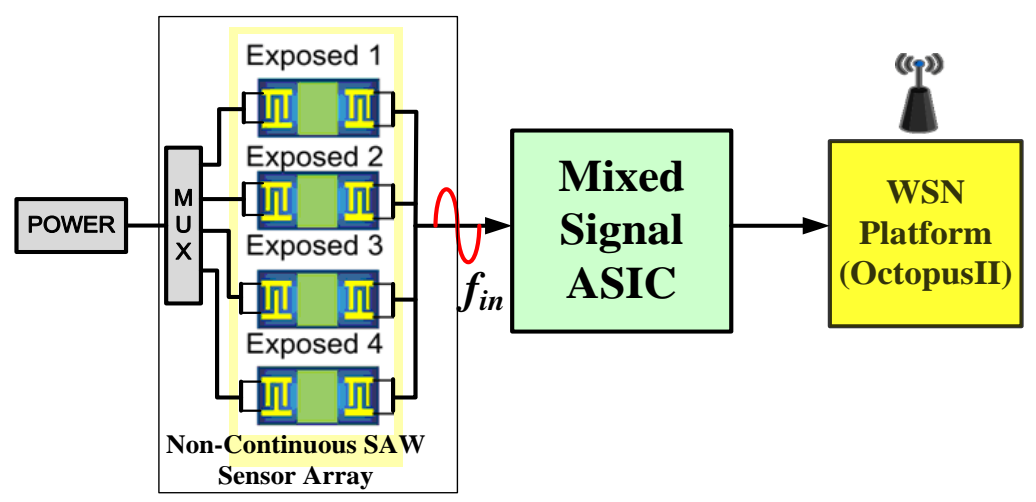

\subsection{Surface Acoustic Wave (SAW) Sensor}

In the late 1970s, various gas sensors were developed based on a variety of operational principles. Compared with other gas sensors, SAW-based gas sensors provide a high degree of sensitivity, reproducibility, and stability [22]. The sensing mechanism is based on the fact that volatile organic compounds are adsorbed on the surface of a substrate and an increase in mass loading causes a shift in frequency. The mechanism behind SAW sensors involves the input of voltage inducing an electric field between the IDTs. The piezoelectric effect induces dynamic strain on the substrate to launch the SAW, propagating across the surface of the substrate. At the receiving end, the IDT converts the mechanical signals to electric signals. Finally, fluctuations in the output frequency are measured. According to the design parameters from the simulation data of delta function model and the cross field model shown in Table 1, $\mathrm{LiNbO}_{3}$-based. SAW devices with IDTs of $\mathrm{Cr} / \mathrm{Au}(20 \mathrm{~nm} / 100 \mathrm{~nm})$ were fabricated in a photolithography process with a center frequency of 117.4 MHz. The size of the SAW sensors was $3 \mathrm{~mm} \times 2 \mathrm{~mm}$.

Table 1. Design parameters of the SAW chip.

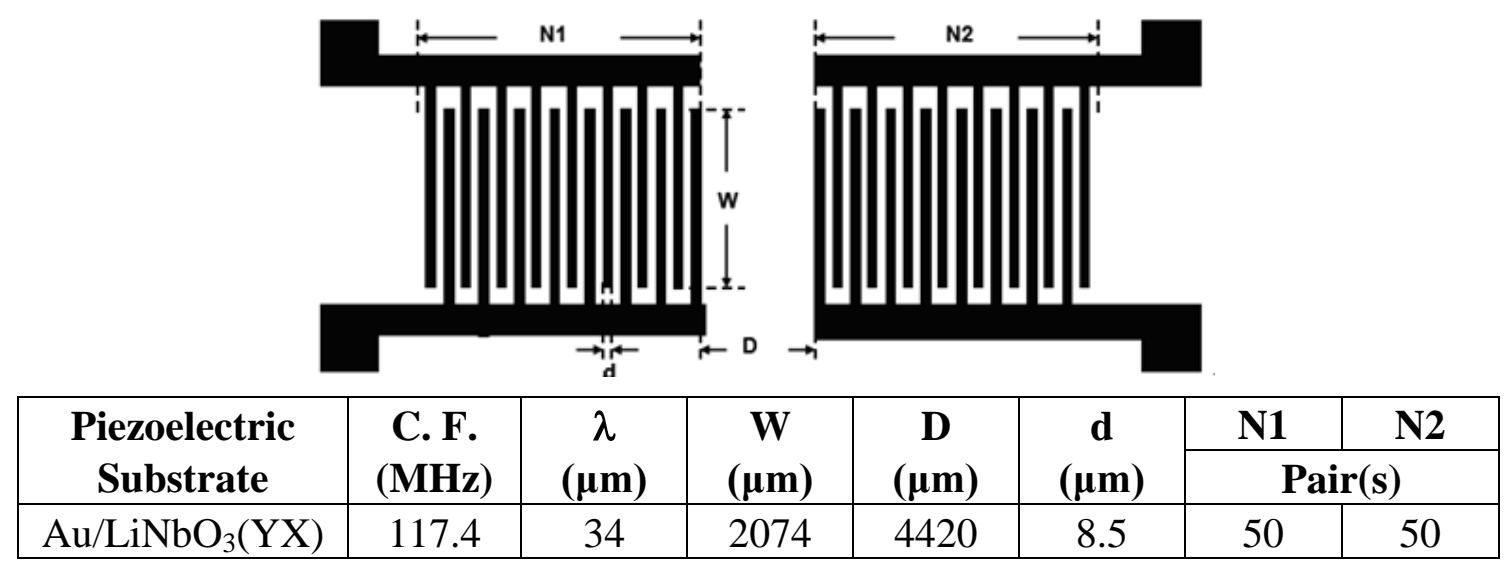


Figure 2. (A) The SAW device was fabricated using the standard photolithography process. (B) An enlarged optical image of interdigital transducers (IDTs). (C) Optical image of the SAW chip.

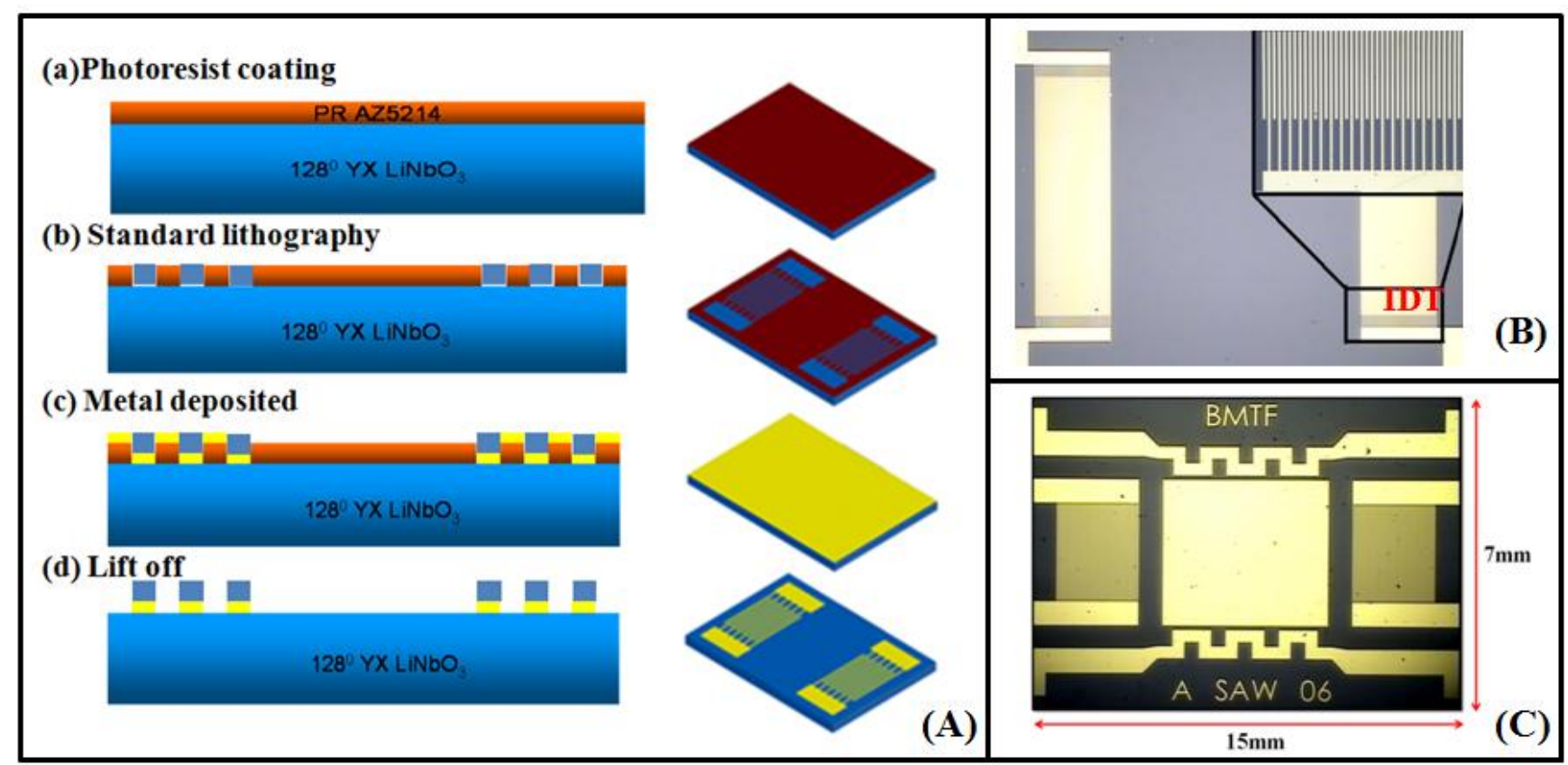

\subsection{SAW Sensor Array}

A SAW array was employed to fabricate a gas sensor with the advantages of small size, low cost, high sensitivity, and rapid response. As shown in Figure 3, the $2 \times 2$ non-continuous working oscillators were controlled by a multiplexing technique as a switching element [23].

The film of the sensor operated like a smart skin, responsible for generating chemical signals from interactions between molecules and the film [24]. The sensing polymer film acted as the mucosa of the nasal cavity, playing an important role in the detection of gases. To enhance sensitivity and selectivity, seven polymers were selected as materials to be used in the sensitive film, spin-coated on the surface of the resonators of the array (Figure 4). These polymers included poly- $N$-vinylpyrrolidone (PNVP), poly-4-vinylphenol (P4VP), polyvinyl acetate (PVAc), polyethylene-glycol (PEG), polystyrene (PS), polystyrene-co-maleic anhydride (PSMA) and polysulfone (Psu).

Figure 3. (A) The schematic array circuit and (B) The photo of a SAW array with $2 \times 2$ non-continuous working oscillators and SAW chips.
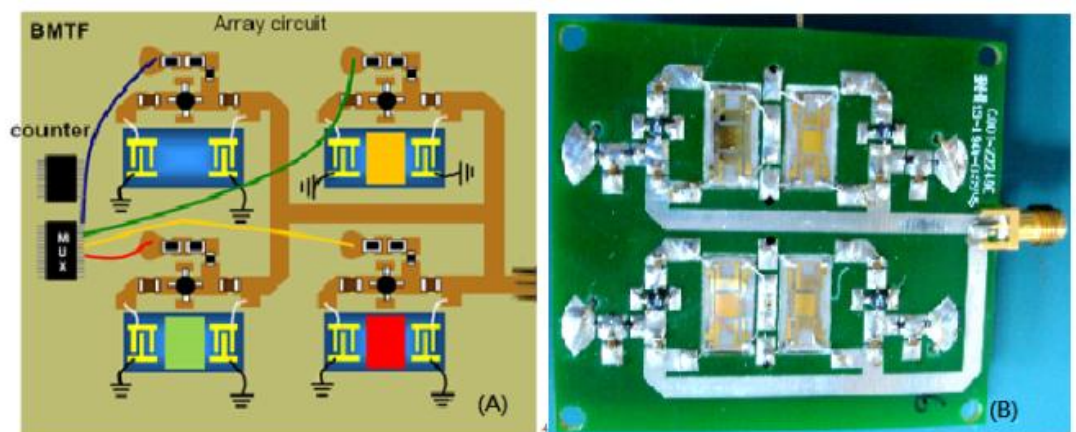
Figure 4. Seven polymer formulas selected as the sensitive films.<smiles>C=CN1CCCC1=O</smiles>

PNVP

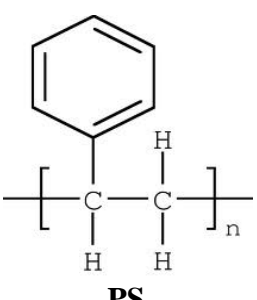

PS<smiles>CC(C)(C)C(c1ccc(O)cc1)C(C)(C)C</smiles>

P4VP

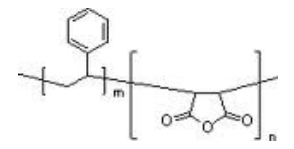

PSMA<smiles>CCC(C)OC(C)=O</smiles>

PVAc<smiles>CC(C)OCCO</smiles>

PEG

In the E-Nose system, a sensor array was used to form patterns for use in the recognition of gases. In this study, the sensor array comprised four SAW sensor devices. These four sensors did not operate at the same time by controlling the oscillators non-continuously to minimize power consumption and interference from crosstalk [23]. In addition, by operating the SAW array non-continuously, all sensor outputs could be connected, thereby only one interface readout circuit was needed, saving chip area and total cost. In this study, the non-continuous SAW sensor reached a stable output within $0.2 \mathrm{~s}$ after being switched on. Therefore, each sensor was switched on for $1 \mathrm{~s}$ to ensure stable sensor output.

\subsection{Mixed Signal Interface ASIC}

There are many kinds of circuit implementations to detect SAW frequencies and/or phases. One way is to convert the SAW frequency into a voltage signal. This approach wastes a large portion of the voltage range because the shift in the frequency of the sensor does not exceed a certain amount [25]. Another way measures phase differences, but the resolution is only at the level of MHz [26]. In this study, the center frequency was at $117.4 \mathrm{MHz}$, and the maximum frequency shift did not exceed $1 \mathrm{MHz}$, representing as little less than $1 \%$ that of the center frequency. Therefore, if a direct frequency to voltage converter was adopted, assuming a linear conversion from frequency to voltage, approximately $99 \%$ of the voltage range was wasted, making it difficult to improve resolution.

In this study, we implemented a mixed-signal interface chip, as shown in Figure 5. The first stage of the chip was analog, including a mixer, low-pass filter, and comparator. The function of the analog stage was to modulate, filter, and convert the signal from the sensor into a square wave. The second stage of the chip was a digital readout to detect changes in sensor frequency. To read out the sensor signal (frequency change), the frequency of the sensor $f_{i n}$ was subtracted from that of a reference sensor $f_{\text {ref. }}$. This resulted in a decrease in the frequency change $f_{\text {ref }}-f_{\text {in }}$ compared to $f_{\text {ref. }}$ Because the amplitude of the signal requiring conversion was smaller, resolution could be improved and power consumption could be reduced. The reference sensor was not coated with a polymer membrane; therefore, the variations in its frequency due to input gas were very small. Nevertheless, the reference sensor was still influenced by the same environment parameters as the sensing sensor, such as 
temperature and humidity. Subtracting $f_{\text {in }}$ from $f_{\text {ref }}$ helped to eliminate the background effect of the sensor.

Figure 5. Block diagram of the mixed-signal interface chip.

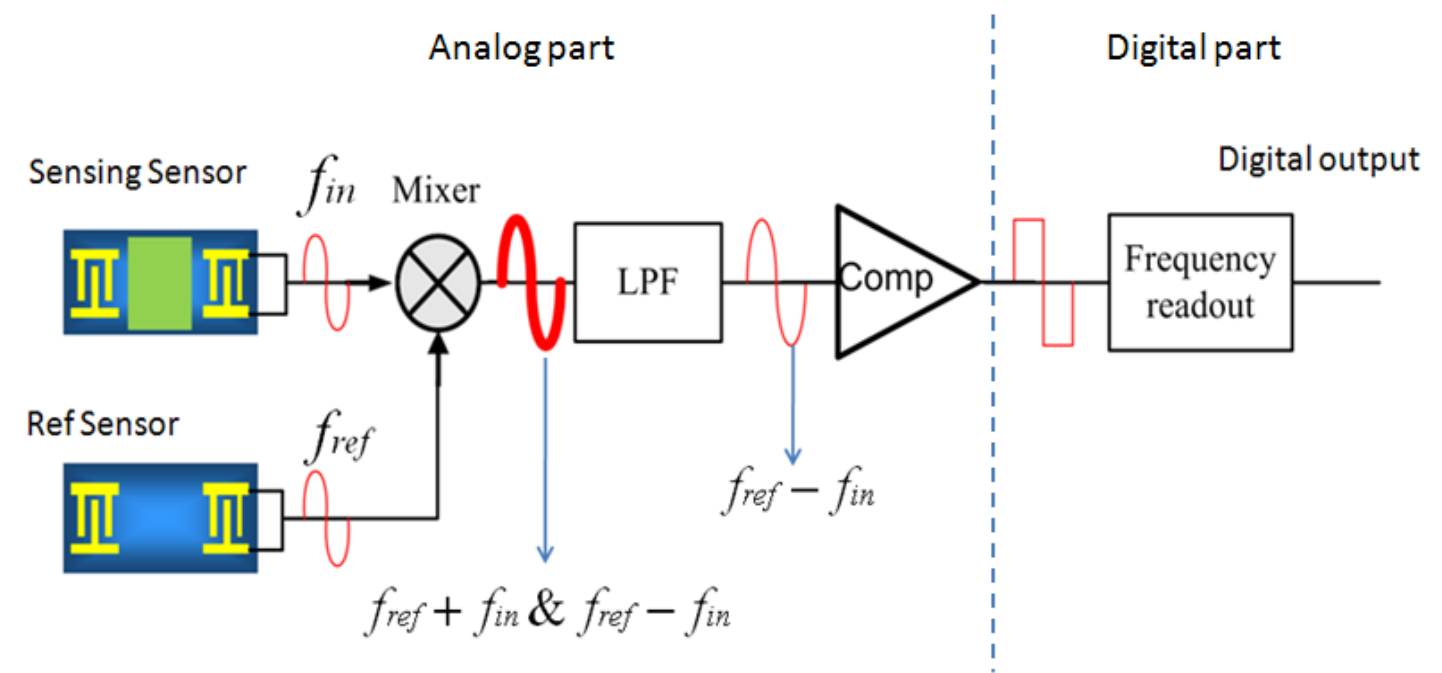

We integrated a mixer into the frontend of the analog section, the output of which had a high frequency term $f_{r e f}+f_{\text {in }}$ and a low frequency term $f_{r e f} f_{\text {in }}$. After the mixer, a low-pass filter passed the low frequency term $f_{r e f}-f_{\text {in }}$, to a comparator to generate a square wave output to the digital stage.

A digital frequency readout circuit received the output from the comparator and reported the frequency data. The main idea was to use three counters with D flip-flops (DFF) for storage, as shown in Figure 6.

Figure 6. Schematic of digital frequency readout circuit.

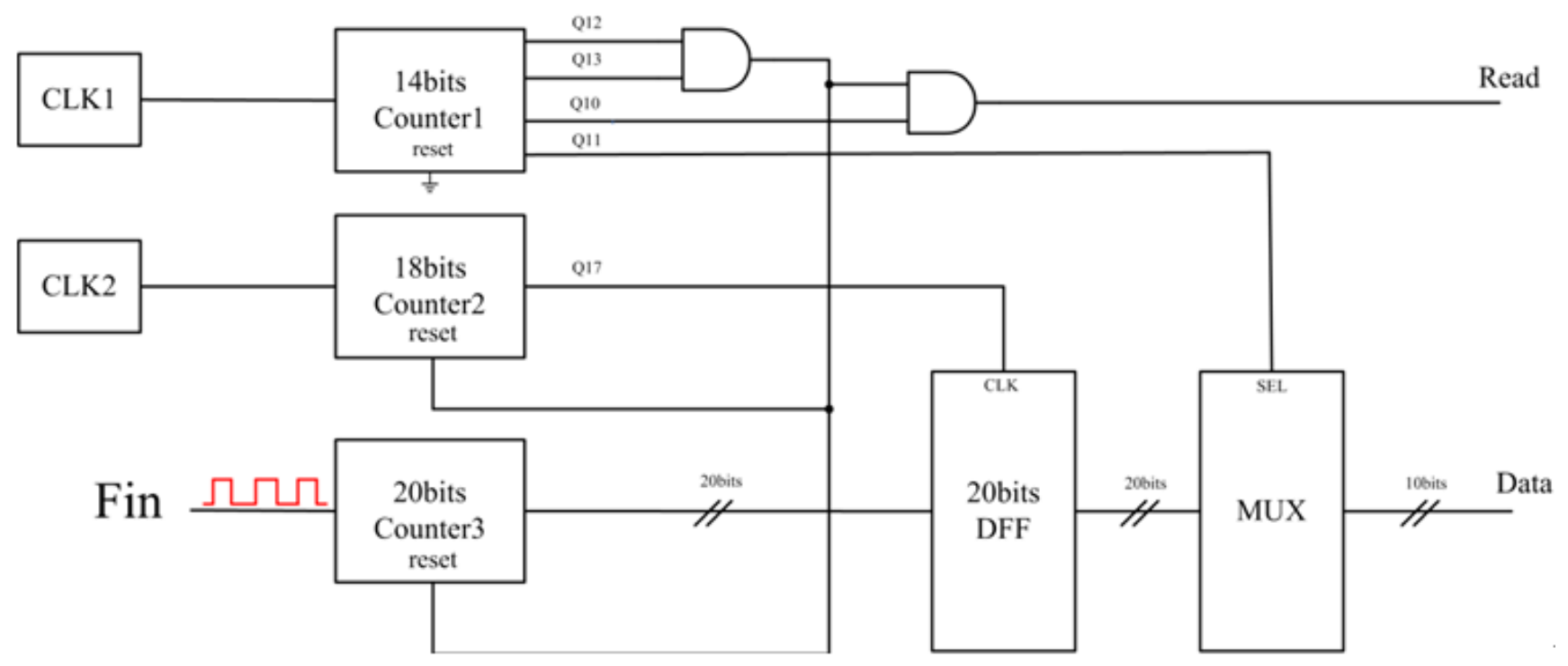

Counter 2 provided a fixed sampling time $\mathrm{T}_{\mathrm{S}}=2^{17} \times \mathrm{T}_{\mathrm{CLK} 2}$, where $\mathrm{T}_{\mathrm{CLK} 2}$ was the clock period of CLK2. The input signal (Fin) went into Counter 3 as the clock. When Counter 3 counted for the time $2^{17} \times \mathrm{T}_{\mathrm{CLK} 2}$, the MSB of Counter 2 triggered the DFF and stored the output data $(D)$ of Counter 3 at 
this moment. Counter 1 generated control signals for the multiplexor (MUX), the reset signal of Counter 3, and the Read signal for the Octopus II. The multiplexor (MUX) output the data stored in the DFF. The input signal (Fin) could be calculated later by:

$$
\mathrm{F}_{\text {in }}=\frac{\mathrm{D}}{2^{17}} \times \mathrm{f}_{\mathrm{CLK} 2}=\frac{\mathrm{D}}{\mathrm{T}_{\mathrm{S}}}
$$

According to Equation (1), the resolution of the mixed-signal interface chip depends on the sampling time $\mathrm{T}_{\mathrm{S}}$. Theoretically, a finer resolution can be achieved by a longer sampling time. For example, a sample time of one second corresponds to a resolution of $1 \mathrm{~Hz}$ (each bit in Counter 3 represents $1 \mathrm{~Hz}$ ), and a sampling time of $100 \mathrm{~ms}$ corresponds to a resolution of $10 \mathrm{~Hz}$ (each bit in Counter 3 represents $10 \mathrm{~Hz}$ ). Since each sensor was switched on for $1 \mathrm{~s}$, a sampling time of $1 \mathrm{~s}$ would be too long. Moreover, $10 \mathrm{~Hz}$ resolution was enough for detection. Consequently, a sampling time of $100 \mathrm{~ms}$ was chosen in this work.

\subsection{WSN Platform}

The Octopus II $[27,28]$ WSN platform was selected to transmit sensing data. Because the output of the frequency readout circuitry was not a fast-changing signal, it could be connected directly to the Octopus 50-pin extension connector. Octopus II includes MSP430F1611, a USB Interface, Inverted F, and SMA Type Antenna. The basic features of Octopus II are as follows: RF range is approximately $450 \mathrm{~m}$, board size is $80 \mathrm{~mm} \times 31 \mathrm{~mm}$, maximum output power is approximately $10 \mathrm{dBm}$, compatible with IEEE 802.15.4 (ZigBee), and operates using $2 \times$ AA batteries (3.3 V 2,700 mAh). Detailed information can be obtained from the website (http://www.wsnc.ntu.edu.tw/Files/ Octopus--_0913_V1_2\%20[----].pdf).

\subsection{Gas Experimental Setup}

The sensor data was transmitted through a wireless module to a personal computer operating as a base station for data storage, processing, and analysis. To verify the E-Nose sensor node, three tests had to be performed: (1) a gas experiment to validate the SAW sensor array; (2) a configuration test to verify the accuracy of the mixed-signal readout chip; (3) a verification test to determine the correctness of the wireless transmission.

Figure 7 illustrates the gas experimental setup, in which seven different sensing membranes were used in the SAW sensor array. The array was tested using ethanol and acetone, and the complete system was tested in a 1L 4-neck bottle chamber. The four windows were for the vacuum pump, test gas input, wire connection, and air flow valve. Considering that the membrane may have absorbed excessive water vapors, we first baked the sensor array for 20 minutes at $90{ }^{\circ} \mathrm{C}$, prior to measurement, to reduce the water vapor interference from inside the membrane. One cycle of gas testing involved four steps:

(1) Pumping: The system was operated stably under vacuum pumping for 5 minutes.

(2) Injection: Following the stabilization of frequency, the vacuum pump was turned off and the air flow valve was closed. Test gas was injected into the chamber, and the device responded for 5 minutes. 
(3) Air: The vacuum pump was turned on and the air flow valve was opened to clean the chamber for 5 minutes.

(4) The air flow valve closed, returning to step (1).

The total time required for one cycle was approximately 15 minutes. The system responded very rapidly to the test gas causing a frequency shift within 3 minutes. To test the accuracy of the mixedsignal readout chip, the output was compared with a frequency counter.

Figure 7. Gas experimental setup.

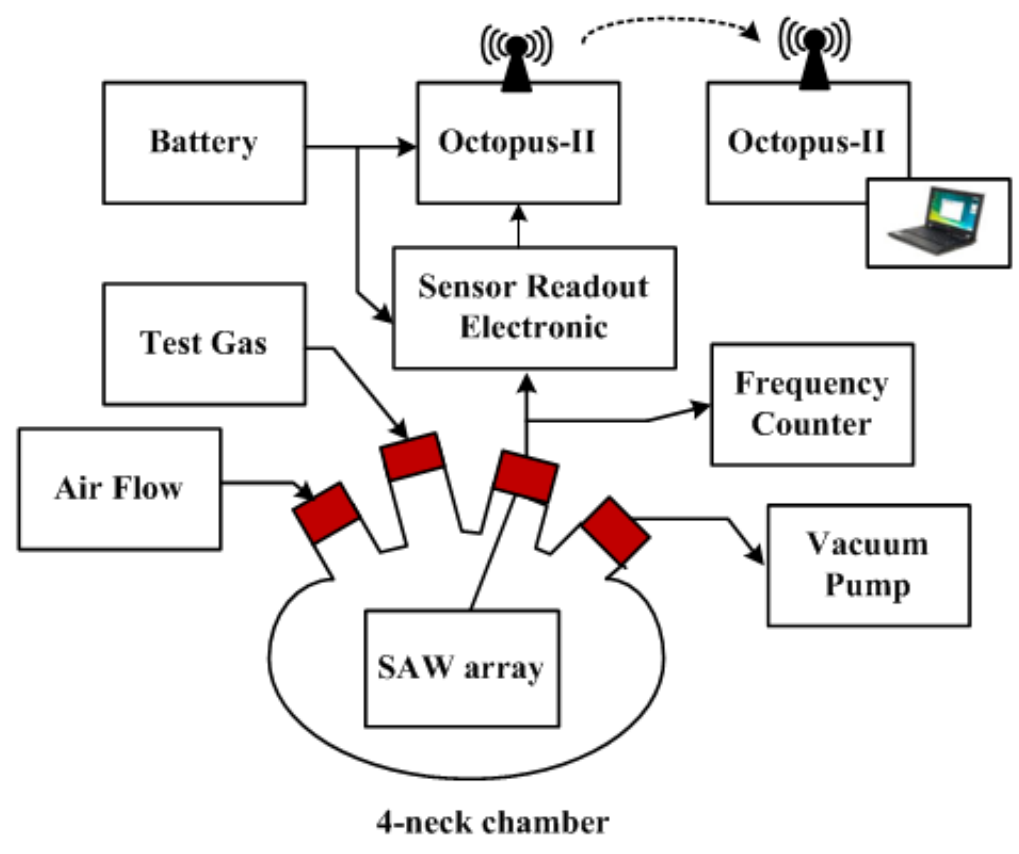

\section{Experimental Results and Discussion}

\subsection{SAW Gas Sensor}

The SAW array was tested using ethanol and acetone. Figure 8 shows a typical response of the sensors (coated with PMSA, PEG, and PNVP) exposed to ethanol. The experiment was repeated three times. The baseline seemed to drift due to temperature variation. The SAW devices are known to be sensitive to its environment parameters, especially temperature [29]. Currently, many SAW devices are manufactured with materials such as lithium niobate or lithium tantalite. The advantage of using these two materials is their high $K^{2}$ values, at the price of their high temperature coefficients. Our way to compensate the temperature effect is to use a reference SAW device without membrane.

\subsection{Sensor Interface Measurement Results}

To verify the accuracy of the interface chip, a signal generator was used to emulate the SAW signals. A spectrum analyzer measured the outputs of the mixer and the low-pass filter. The two input signals to the mixer were $117 \mathrm{MHz}$ and $118 \mathrm{MHz}$ sinusoidal wave with amplitude $0.5 \mathrm{~V}$. As a result, the spectrum analyzer showed that the mixer output spectrum had two frequency components, $1 \mathrm{MHz}$ 
and $235 \mathrm{MHz}$, and the low-pass filter output spectrum was $1 \mathrm{MHz}$. These results verified the function of the mixer and the low-pass filter.

Figure 8. Typical sensor response (gas: ethanol, membrane: PNVP).

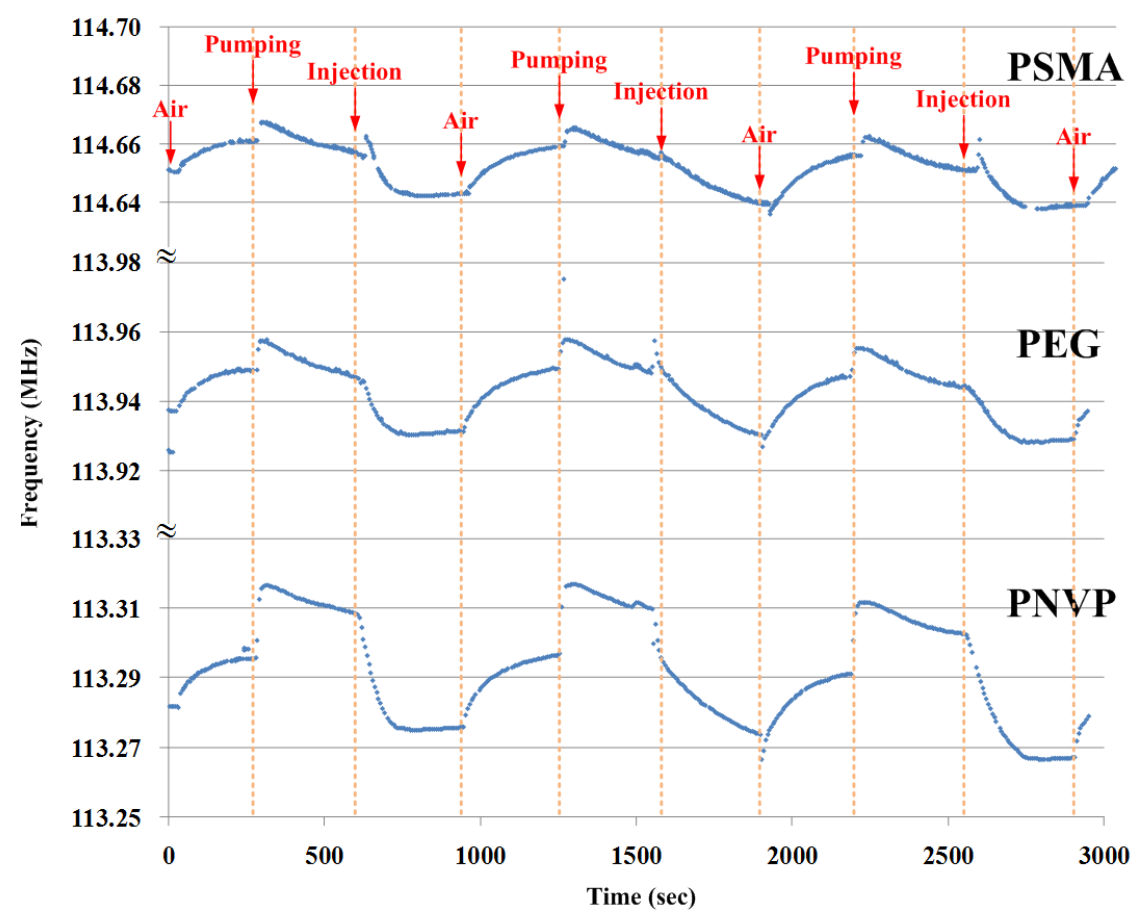

The output of the low-pass filter was sent to a comparator to convert the signal into a square wave, which was passed to the digital frequency readout circuit. To test the frequency readout circuit, a square wave was input to the circuit and into a frequency counter at the same time. The test frequencies were 10, 100, 1 k, 10 k, $100 \mathrm{k}$, and $1 \mathrm{MHz}$. Each frequency was sampled 1,000 times. The total test time was 100 seconds. In all the test results, the frequency readout circuit outputs were the same as the frequency counter. The power consumption for the entire chip was $1.48 \mathrm{~mW}$ using a power supply of 3.3 V. Figure 9 shows a photo of the die used in this low-power mixed-signal SAW interface ASIC. Table 2 provides a benchmark with other studies of the SAW interface circuit $[25,26]$.

Table 2. Benchmark with other works of SAW interface circuit.

\begin{tabular}{lccc}
\hline & {$[25]$} & {$[26]$} & This Work \\
\hline Year & 2005 & 2000 & 2010 \\
Supply Voltage & $3.3 \mathrm{~V}$ & $2.5 \mathrm{~V}$ & $3.3 \mathrm{~V}$ \\
Process Technology & $0.35 \mu \mathrm{m}$ & $\mathrm{GaAs}$ & $0.18 \mu \mathrm{m}$ \\
Power Consumption & $38.35 \mathrm{~mW}$ & $225 \mathrm{~mW}$ & $1.48 \mathrm{~mW}$ \\
Resolution & $10 \mathrm{MHz}$ & $3 \mathrm{MHz}$ & $10 \mathrm{~Hz}$ \\
Input Frequency & $354 \mathrm{MHz}$ & $690 \mathrm{MHz}$ & $117.4 \mathrm{MHz}$ \\
\hline
\end{tabular}


Figure 9. Die photo of this low-power mixed-signal SAW interface ASIC.

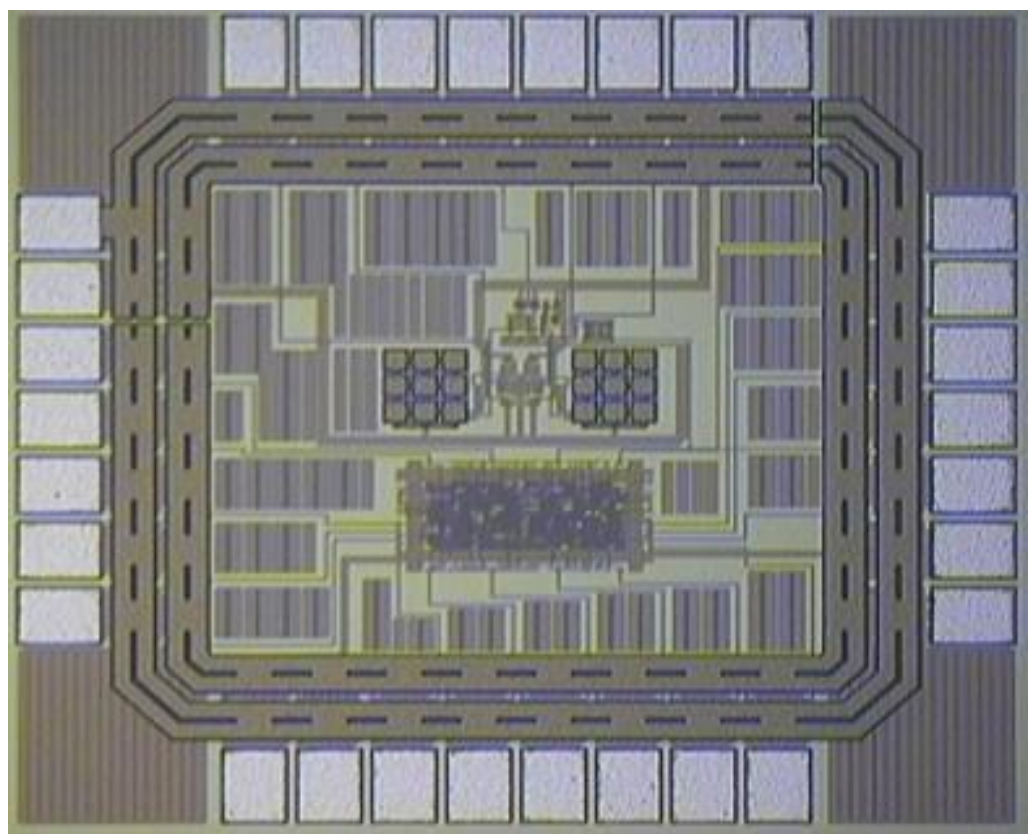

\subsection{Sensor Node}

Figure 10 shows the test setup of the sensor node. The SAW array was connected to the interface chip, and the output was connected to the wireless module. Both the interface chip and the wireless module were running on two $1.5 \mathrm{~V}$ batteries. The wireless module transmitted the sensor data to a base station PC. This data was compared with the frequency counter output according to Equation (2):

$$
\left(\sum_{\mathrm{i}=1}^{\mathrm{n}} \mathrm{A}_{\mathrm{i}}-\mathrm{B}_{\mathrm{i}}\right) / \mathrm{n}=\text { error }
$$

where data list $\mathrm{A}$ is the frequency counter output, data list $\mathrm{B}$ is the data transmitted to the base station $\mathrm{PC}, \mathrm{n}$ is the data number used for comparison. Table 3 summarizes the mean error and standard deviation of the two data lists. Both the mean error and standard deviation between the transmitted data from the sensor node and the frequency counter output are less than $4 \mathrm{~Hz}$.

Table 3. Mean error and standard deviation of the two lists of data.

\begin{tabular}{|c|c|c|c|c|}
\hline \multirow{2}{*}{$\begin{array}{c}\text { Readout data } \\
(\mathrm{Hz})\end{array}$} & \multicolumn{2}{|c|}{ Ethanol } & \multicolumn{2}{c|}{ Acetone } \\
\cline { 2 - 5 } Membrane & & & & \\
\hline PNVP & & 2.56 & 2.41 & 2.47 \\
PS & 2.18 & 3.24 & 1.24 & 1.89 \\
PSMA & 2.47 & 3.39 & 2.17 & 2.52 \\
PEG & 2.48 & 3.25 & 1.25 & 1.44 \\
P4VP & 2.13 & 3.54 & 0.74 & 0.94 \\
PVAc & 2.31 & 2.69 & 1.23 & 1.85 \\
PSu & 1.53 & 2.58 & 3.68 & 2.94 \\
\hline
\end{tabular}


Figure 10. Test setup of the sensor node.

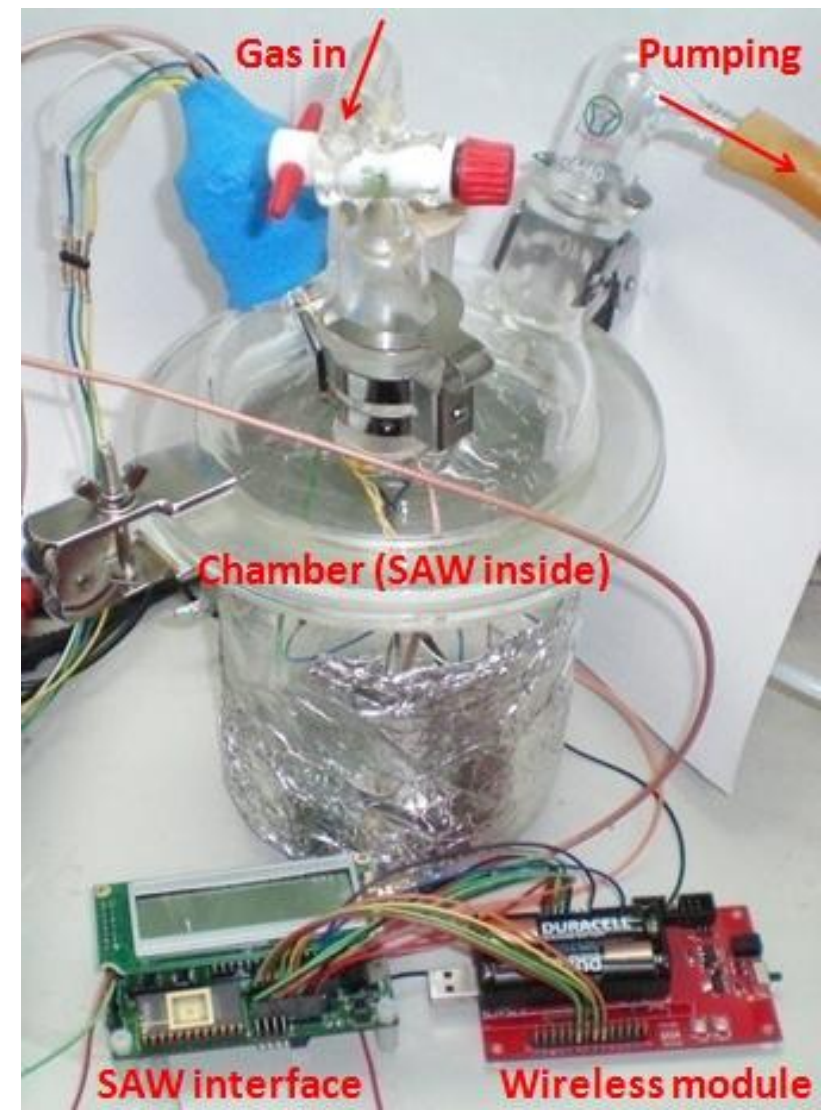

\section{Conclusions}

We have reported a low-cost sensor node comprising a SAW sensor array, an interface chip, and wireless transmission module (Octopus II). The SAW sensor array comprised four SAW sensors with different sensing membranes operating non-continuously. The interface chip provided resolution in the frequency readout as low as $10 \mathrm{~Hz}$. The wireless module transmitted sensor data to a remote computer for storage and analysis. This compact sensor node achieved high resolution, low power consumption, and is suitable for mass production and wireless sensor network applications.

\section{Acknowledgements}

The authors would like to acknowledge the support of the National Science Council of Taiwan, under Contract No. NSC 98-2220-E-007-017 and NSC98-2218-E-007-019. We thank Da-Jeng Yao for the SAW structure, Chia-Min Yang for the polymers, and Jang-Ping Sheu for the Octopus II module. The authors would like to thank National Chip Implementation Center (CIC) for chip fabrication.

\section{References}

1. Buratti, C.; Conti, A.; Dardari, D.; Verdone, R. An overview on wireless sensor networks technology and evolution. Sensors 2009, 9, 6869-6896.

2. Akyildiz, I.F.; Su, W.; Sankarasubramaniam, Y.; Cayirci, E. A survey on sensor networks. IEEE Comm. Mag. 2002, 40, 102-114. 
3. Corchado, J.M.; Bajo, J.; Tapia, D.I.; Abraham, A. Using heterogeneous wireless sensor networks in a telemonitoring system for healthcare. IEEE Trans. Inform. Tech. Biomed. 2010, 14, 234-240.

4. Gungor, V.C.; Hancke, G.P. Industrial wireless sensor networks: Challenges, design principles, and technical approaches. IEEE Trans. Consum. Electron. 2009, 56, 4258-4265.

5. López, J.A.; Soto, F.; Sánchez, P.; Iborra, A.; Suardiaz, J.; Vera, J.A. Development of a sensor node for precision horticulture. Sensors 2009, 9, 3240-3255.

6. Werner-Allen, G.; Lorincz, K.; Ruiz, M.; Marcillo, O.; Johnson, J.; Lees, J.; Welsh, M. Deploying a wireless sensor network on an active volcano. IEEE Inter. Comput. 2006, 10, 18-25.

7. Szewczyk, R.; Osterweil, E.; Polastre, J.; Hamilton, M.; Mainwaring, A.; Estrin, D. Habitat monitoring with sensor networks. Commun. ACM 2004, 47, 34-40.

8. Lee, D. Wireless and powerless sensing node system developed for monitoring motors. Sensors 2008, $8,5005-5022$.

9. Cai, C.J.; Geng, F.H.; Tie, X.X.; Yu, Q.; Peng, L.; Zhou, G.Q. Characteristics of ambient volatile organic compounds (VOCs) measured in Shanghai, China. Sensors 2010, 10, 7843-7862.

10. Gardner, J.W.; Bartlett, P.N.; Dodd, G.H.; Shurmer, H.V. Pattern Recognition in the Warwick Electronic Nose. In Proceedings of 8th International Congress of European Chemoreception Research Organisation, Coentry, UK, 18-22 July 1988.

11. Lazar, Z.; Fens, N.; van der Maten, J.; van der Schee, M.P.; Wagener, A.H.; de Nijs, S.B.; Dijkers, E.; Sterk, P.J. Electronic nose breathprints are independent of acute changes in airway caliber in asthma. Sensors 2010, 10, 9127-9138.

12. Moularat, S.; Robine, E.; Ramalho, O.; Oturan, M.A. Detection of fungal development in a closed environment through the identification of specific VOC: Demonstration of a specific VOC fingerprint for fungal development. Sci. Total Environ. 2008, 407, 139-146.

13. Tang, K.T.; Chiu, S.W.; Pan, C.H.; Hsieh, H.Y.; Liang, Y.S.; Liu, S.C. Development of a portable electronic nose system for the detection and classification of fruity odors. Sensors 2010, 10, 9179-9193.

14. Wen, W.; Shitang, H.; Shunzhou, L.; Minghua, L.; Yong, P. Enhanced sensitivity of SAW gas sensor coated molecularly imprinted polymer incorporating high frequency stability oscillator. Sens. Actuat. B 2007, 125, 422-427.

15. Alizadeh, T.; Zeynali, S. Electronic nose based on the polymer coated SAW sensors array for the warfare agent simulants classification. Sens. Actuat. B 2008, 129, 412-423.

16. Casalnuovo, S.A.; Hietala, V.M.; Heller, E.J.; Frye-Mason, G.C.; Baea, A.G.; Wendt, J.R. Monolithic Integration of GaAs SAW Chemical Microsensor Arrays and Detection Electronics. In Proceedings of Solid-State Sensor and Actuator Workshop, Hilton Head, SC, USA, 4-8 June 2000.

17. Hao, H.C.; Tang, K.T.; Ku, P.H.; Chao, J.S.; Li, C.H.; Yang, C.M.; Yao, D.J. Development of a portable electronic nose based on chemical surface acoustic wave array with multiplexed oscillator and readout electronics. Sens. Actuat. B 2010, 146, 545-553.

18. Lu, S.; Huang, X.; Cui, L.; Zhao, Z.; Li, D. Design and implementation of an ASIC-based sensor device for WSN applications. IEEE Trans. Consum. Electron. 2009, 55, 1959-1967.

19. Harnett, C.K. Open wireless sensor network telemetry platform for mobile phones. IEEE Sens. J. 2010, 10, 1083-1084. 
20. Sun, L.; Crooks, R.M.; Ricco, A.J. Molecular interactions between organized, surface-confined monolayers and vapor-phase probe molecules. 5. acid-base interactions. Langmuir 1993, 9, 1775-1780.

21. Wang, C.; He, X.W.; Chen, L.X. A piezoelectric quartz crystal sensor array self assembled calixarene bilayers for detection of volatile organic amine in gas. Talanta 2002, 57, 1181-1188.

22. Drafts, B. Acoustic wave technology sensors. IEEE Trans. Microw. Theor. Tech. 2001, 49, 795-802.

23. Rapp, M.; Reibel, J.; Voigt, A.; Balzer, M.; Bülow, O. New miniaturized SAW-sensor array for organic gas detection driven by multiplexed oscillators. Sens. Actuat. B 2000, 65, 169-172.

24. Dermody, D.L.; Crooks, R.M.; Kim, T. Interactions between organized, surface-confined monolayers and vapor-phase probe molecules. 11. Synthesis, characterization, and chemical sensitivity of self-assembled polydiacetylene/calix[n]arene bilayers. J. Am. Chem. Soc. 1996, 118, 11912-11917.

25. Yasin, F.M.; Tye, K.F.; Reaz, M.B.I. Design and Implementation of Interface Circuitry for CMOS-based SAW Gas Sensors. In Proceedings of 2005 IEEE International SOC Conference, Herndon, VA, USA, 19-23 September 2005; pp. 161-164.

26. Casalnuovo, S.A.; Hietala, V.M.; Heller, E.J.; Frye-Mason, G.C.; Baea, A.G.; Wendt, J.R. Monolithic Integration of GaAs SAW Chemical Microsensor Arrays and Detection Electronics. In Proceedings of Solid-State Sensor and Actuator Workshop, Hilton Head, SC, USA, 4-8 June 2000.

27. Sheu, J.P.; Chang, C.J.; Sun, C.Y.; Hu, W.K. WSNTB: A Testbed for Heterogeneous Wireless Sensor Networks. In Proceedings of 2008 First IEEE International Conference on Ubi-Media Computing, Lanzhou, China, 15-16 July 2008; pp. 338-343.

28. Sheu, J.P.; Chen, P.C.; Hsu, C.S. A distributed localization scheme for wireless sensor networks with improved grid-scan and vector-based refinement. IEEE Trans. Mob. Comput. 2008, 7 , 1110-1123.

29. Wolff, U.; Dickert, F.L.; Fischerauer, G.K., Greibl, W.; Ruppel, C.C.W. SAW sensors for harsh environments. IEEE Sens. J. 2001, 1, 4-13.

(C) 2011 by the authors; licensee MDPI, Basel, Switzerland. This article is an open access article distributed under the terms and conditions of the Creative Commons Attribution license (http://creativecommons.org/licenses/by/3.0/) 